\title{
Four-Dimensional Regularization for Electrical Impedance Tomography Imaging
}

\author{
Tao Dai ${ }^{1}$, Manuchehr Soleimani ${ }^{2}$, Andy Adler ${ }^{1}$ \\ ${ }^{1}$ Systems and Computer Engineering, Carleton University, Ottawa, Canada \\ William Lee Innovation Centre, University of Manchester, U.K.
}

\begin{abstract}
This paper proposes 4-D EIT image reconstruction for functional EIT measurements. The approach directly accounts for 3-D interslice spatial correlations and temporal correlations between images in successive data frames. Image reconstruction is posed in terms of an augmented image $\tilde{x}$ and measurement vector $\tilde{\mathbf{y}}$, which concatenate the values from the $d$ previous and future frames. Image reconstruction is then based on an augmented regularization matrix $\tilde{\mathbf{R}}$, which accounts for a model with 4-D correlations of image elements, interslices and temporal frames. The temporal correlation matrix is objectively calculated from measurement data. Results of simulations are compared by reconstruction algorithms based on conventional 3-D and proposed 4-D priors.
\end{abstract}

Keywords - Electrical Impedance Tomography, regularization, spatial and temporal priors, image reconstruction.

\section{INTRODUCTION}

Electrical Impedance Tomography (EIT) calculates an estimate of the conductivity distribution within a body based on current stimulations and voltage measurements on the body surface. EIT typically has poor spatial resolution, but can have excellent temporal resolution; some recent systems have frame rates up to 1000/s [1]. Such high temporal resolution makes EIT a promising technology to monitor fast physiological events which affect the conductivity distribution. e.g. cardiac activities, high frequency ventilation, functional brain EIT.

3-D EIT algorithms use multi-plane electrode arrangements to accurately reconstruct $3-\mathrm{D}$ conductivity distributions [2][3]. In 3-D EIT reconstructions, the Tikhonov [4] and the NOSER priors [3] are commonly used regularization approaches. While such priors are easy to compute, they assume that elements of the conductivity distributions are statistically independent - which is clearly not true for most EIT applications. Instead, conductivity distributions are likely to change relatively slowly, both in the spatial and time directions.

Most EIT reconstruction algorithms solve data frames independently, although Kalman filter algorithms track the image changes across frames. Formulated as an iterative state estimation problem, the first Kalman filter based algorithm for difference EIT was proposed in [5].
In this paper, we explore an approach to describe spatial and temporal correlations in the images in the regularization prior. The correlations between successive vertial slices in a 3-D phantom are modelled with a parameter $\eta$, and the correlations between successive data frames (ie. 4-D) are modelled with a parameter $\gamma$. We then propose an image reconstruction algorithm which reconstructs the 3-D image at frame $t$ from the set of data in a window of frames from $t-d$ to $t+d$.

\section{Methods}

We consider an EIT system with $n_{E}$ electrodes applied to a body using planar placement [3] and adjacent current stimulation with parallel voltage measurement. $n_{E}$ current stimulation patterns are sequentially applied and $n_{V}$ differential measurements are made for each stimulation. Difference EIT calculates difference data $\mathbf{y},\left([\mathbf{y}]_{i}=\left[\mathbf{v}_{2}\right]_{i}-\left[\mathbf{v}_{1}\right]_{i}\right.$; or normalized as $\left.[\mathbf{y}]_{i}=\left(\left[\mathbf{v}_{2}\right]_{i}-\left[\mathbf{v}_{1}\right]_{i}\right) /\left[\mathbf{v}_{1}\right]_{i}\right)$. To improve its precision, $\mathbf{v}_{1}$ is typically averaged over many data frames, at a time when the conductivity distribution may be assumed to be stable; we thus assume that $\mathbf{v}_{1}$ is noise free.

The body under investigation is modelled using a cylindrical finite element model (FEM) which has $n_{N}$ piecewise smooth tetrahedral elements and is discretized onto $k$ slices, represented by a vector $\boldsymbol{\sigma} \in \mathbb{R}^{n_{N}}$ (In this paragraph, $\sigma$ represents conductivity; elsewhere in this paper, $\sigma$ is the standard deviation). Again, difference EIT calculates a vector of conductivity change, $\mathbf{x}=\sigma_{2}-\sigma_{1}$ between the present conductivity distribution, $\sigma_{2}$, and the reference measurement, $\sigma_{1}$. For small variations around $\sigma_{1}$, the relationship between $\mathbf{x}$ and $\mathbf{y}$ can be linearized (giving the difference EIT forward model):

$$
\mathbf{y}=\mathbf{J} \mathbf{x}+\mathbf{n}
$$

where $\mathbf{J} \in \mathbb{R}^{n_{M} \times n_{N}}$ is the Jacobian or sensitivity matrix; $\mathbf{n} \in \mathbb{R}^{n_{M}}$ is the measurement noise which is assumed to be uncorrelated white Gaussian. $\mathbf{J}$ is calculated from the FEM as $\mathbf{J}_{i j}=\left.\frac{\partial \mathbf{y}_{i}}{\partial \mathbf{x}_{j}}\right|_{\boldsymbol{\sigma}_{1}}$. This system is underdetermined since $n_{N}>n_{M}$. This problem is solved using regularization techniques (e.g. [6], [7]) to calculate a conductivity change 
estimate, $\hat{\mathbf{x}}$, which is both faithful to the measurements, $\mathbf{y}$, and to a priori constraints on a "reasonable" image.

In subsequent sections we consider the following reconstruction approaches: 1) Gauss-Newton (GN) inverse, using $\mathbf{y}_{t}$ only; 2) Temporal inverse, using $\mathbf{y}_{t-d} \cdots \mathbf{y}_{t+d}$ based on a temporal prior model; and 3) 4-D prior inverse, using $\mathbf{y}_{t-d} \cdots \mathbf{y}_{t+d}$ based on the temporal prior model and 3-D interslice spatial model.

\section{A. One-step linear Gauss-Newton (GN) solver}

The GN inverse seeks a solution, $\hat{\mathbf{x}}$, which minimizes

$$
\|\mathbf{y}-\mathbf{J} \hat{\mathbf{x}}\|_{\Sigma_{n}^{-1}}^{2}+\left\|\mathbf{x}-\mathbf{x}^{\circ}\right\|_{\Sigma_{x}^{-1}}^{2}
$$

where $\mathrm{x}^{\circ}$ represents the expected value of element conductivity changes, which is zero for difference EIT. $\Sigma_{n} \in$ $\mathbb{R}^{n_{M} \times n_{M}}$ is the covariance matrix of the measurement noise $\mathbf{n}$. Since $\mathbf{n}$ is uncorrelated, $\Sigma_{n}$ is a diagonal matrix with $\left[\Sigma_{n}\right]_{i, i}=\sigma_{i}^{2}$, where $\sigma_{i}^{2}$ is the noise variance at measurement $i$. $\Sigma_{x} \in \mathbb{R}^{n_{N} \times n_{N}}$ is the expected image covariance.

Let $\mathbf{W}=\sigma_{n}^{2} \Sigma_{n}^{-1}$ and $\mathbf{R}=\sigma_{x}^{2} \Sigma_{x}^{-1} . \mathbf{W}$ and $\mathbf{R}$ are heuristically determined from a priori considerations. Here $\sigma_{n}$ is the average measurement noise amplitude and $\sigma_{x}$ is the a priori amplitude of conductivity change. $\mathbf{W}$ models the measurement accuracy. For uncorrelated noise, each diagonal element is proportional to the signal to noise ratio. For difference EIT with identical channels, $\mathbf{W}=\mathbf{I}$. The regularization matrix $\mathbf{R}$ may be understood to model the "unlikelihood" of image elements correlations.

By solving (2) and defining the hyperparameter $\lambda=$ $\sigma_{n} / \sigma_{x}$, a linearized, one-step inverse solution is obtained

$$
\hat{\mathbf{x}}=\left(\mathbf{J}^{T} \mathbf{W} \mathbf{J}+\lambda^{2} \mathbf{R}\right)^{-1} \mathbf{J}^{T} \mathbf{W} \mathbf{y}=\mathbf{B y}
$$

where $\mathbf{B}=\left(\mathbf{J}^{T} \mathbf{W} \mathbf{J}+\lambda^{2} \mathbf{R}\right)^{-1} \mathbf{J}^{T} \mathbf{W}$ is the linear, onestep inverse. $\lambda$ controls the trade-off between resolution and noise attenuation in the reconstructed image.

If image elements are assumed to be independent with identical expected magnitude, $\mathbf{R}$ becomes an identity matrix I and (3) uses zeroth-order Tikhonov regularization. For EIT, such solutions tend to push reconstructed noise toward the boundary, since the measured data is much more sensitive to boundary image elements. Instead, $\mathbf{R}$ may be scaled with the sensitivity of each element, so that $\mathbf{R}$ is a diagonal matrix with elements $[\mathbf{R}]_{i, i}=\left[\mathbf{J}^{T} \mathbf{J}\right]_{i, i}^{p}$. This is the NOSER prior of [6] for an exponent $p$.

In this paper, the NOSER prior is used to calculate the matrix $\mathbf{R}$ with $p=0.5$ in all tested algorithms. The choice of exponent is a heuristic compromise between the pushing noise to the boundary $(p=0)$ or to the centre $(p=1)$.
In (3), the term inverted is of size $n_{N} \times n_{N}$. We rewrite the matrix $\mathbf{B}$ using the data form [8] as:

$$
\mathbf{B}=\mathbf{P} \mathbf{J}^{T}\left(\mathbf{J} \mathbf{P} \mathbf{J}^{T}+\lambda^{2} \mathbf{V}\right)^{-1}
$$

where $\mathbf{P}=\mathbf{R}^{-1}=\Sigma_{x} / \sigma_{x}^{2}$ and $\mathbf{V}=\mathbf{W}^{-1}=\Sigma_{n} / \sigma_{n}^{2}$; Using (4), the size of the term in the inverse is reduced to $n_{M} \times n_{M}$. This is especially significant for 3-D EIT models and the temporal inverse which will be introduced below.

\section{B. 3-D spatial prior with interslice correlation}

In this section, we develop a model of a 3-D prior $(\mathbf{P})$ which accounts for a priori correlations between image elements. We only account for those correlations in the vertical direction, although the approach can be directly extended to account for both horizontal and vertical correlations.

The prior, $\mathbf{P}$, is designed in the form

$$
\mathbf{P}=\mathbf{P}_{N}^{\frac{1}{2}} \mathbf{P}_{C} \mathbf{P}_{N}^{\frac{1}{2}}
$$

where $\mathbf{P}_{N}$ is a diagonal matrix and reflects the weightings of the NOSER prior. Rather than calculate $\mathbf{R}$ and then its inverse, we directly compute $\mathbf{P}_{N}^{\frac{1}{2}}$ using

$$
\left[\mathbf{P}_{N}^{\frac{1}{2}}\right]_{i, i}=\left[\mathbf{J}^{T} \mathbf{J}\right]_{i, i}^{-\frac{p}{2}}
$$

$\mathbf{P}_{C}$ is designed to account for the interslice correlations. Its value is zero, except for elements which are in the same vertical column, in which case has the weighting $\eta^{|d|}$ where $d$ is the interslice distance. Thus,

$$
\left[\mathbf{P}_{C}\right]_{i, j}= \begin{cases}\eta^{\left|S_{i}-S_{j}\right|} & \text { if } X_{i}=X_{j} \text { and } Y_{i}=Y_{j} \\ 0 & \text { otherwise. }\end{cases}
$$

where $\left(X_{i}, Y_{i}\right)$ is the horizontal $(x, y)$ centre position of element $i$, and $S_{i}$ is the vertical layer number. This formulation is applicable for vertically extruded FEM meshes.

\section{Temporal solver}

In this section, we develop a temporal image reconstruction algorithm which calculates the image at a current frame using a set of data frames nearby in time. This differs from Kalman filter based algorithms, since the data frame sequence is treated as a single inverse problem, with a regularization prior to account for both spatial and temporal correlations between image elements. Given a vertically concatenated sequence of measurements frames $\tilde{\mathbf{y}}=\left[\mathbf{y}_{-d} ; \ldots ; \mathbf{y}_{0} ; \ldots ; \mathbf{y}_{d}\right]$ and the corresponding concatenated images $\tilde{\mathbf{x}}=\left[\mathbf{x}_{-d} ; \ldots ; \mathbf{x}_{0} ; \ldots ; \mathbf{x}_{d}\right]$, the direct temporal forward model (1) is rewritten as

$$
\tilde{\mathbf{y}}=\tilde{\mathbf{J}} \tilde{\mathbf{x}}+\tilde{\mathbf{n}}
$$


where $\tilde{\mathbf{n}}=\left[\mathbf{n}_{-d} ; \ldots ; \mathbf{n}_{0} ; \ldots ; \mathbf{n}_{d}\right]$. Based on the approximation that $\mathbf{J}$ is constant, $\tilde{\mathbf{J}}=\mathbf{I} \otimes \mathbf{J}$, where the identity matrix $\mathbf{I}$ has size $2 d+1$, and $\otimes$ is the Kronecker product.

The correlation of corresponding elements between adjacent frames (delay is 1) can be evaluated by an interframe correlation $\gamma$ which has value between 0 (independent) and 1 (fully dependent). $\gamma$ could possibly be negative if subsequent frames have inverse correlation, although this scenario is physiologically unrealistic. As frames become separated in time, the interframe correlation decreases; for a separation $\delta$, the interframe correlation is assumed as $\gamma^{\delta}$. Frames with large time lag, $|\delta|>d$, can be considered independent. The one-step inverse (4) for image reconstruction then becomes

$$
\tilde{\mathbf{B}}=\tilde{\mathbf{P}} \tilde{\mathbf{J}}^{T}\left(\tilde{\mathbf{J}} \tilde{\mathbf{P}} \tilde{\mathbf{J}}^{T}+\lambda^{2} \tilde{\mathbf{V}}\right)^{-1}
$$

where $\tilde{\mathbf{V}}=\mathbf{I} \otimes \mathbf{V}$. $\tilde{\mathbf{V}}$ is diagonal since measurement noise is uncorrelated between frames. $\tilde{\mathbf{P}}=\boldsymbol{\Gamma} \otimes \mathbf{P}$, where $\boldsymbol{\Gamma}$ is the temporal weight matrix of an image sequence $\tilde{\mathbf{x}}$ and is defined to have the form as

$$
[\boldsymbol{\Gamma}]_{i, j}=\gamma^{|i-j|} \quad i, j=1,2, \ldots, 2 d+1 .
$$

From (9) and (10),

$$
\tilde{\mathbf{B}}=\left[\boldsymbol{\Gamma} \otimes\left(\mathbf{P} \mathbf{J}^{T}\right)\right]\left[\boldsymbol{\Gamma} \otimes\left(\mathbf{J P J}^{T}\right)+\lambda^{2}(\mathbf{I} \otimes \mathbf{V})\right]^{-1}
$$

In this case, the one step solution for the current image $(\hat{\mathbf{x}})$ is rewritten as

$$
\hat{\mathbf{x}}_{0}=\tilde{\mathbf{B}}_{0} \tilde{\mathbf{y}}
$$

where $\tilde{\mathbf{B}}_{0}$ is the rows $n_{M} d+1 \ldots n_{M}(d+1)$ of $\tilde{\mathbf{B}}$.

\section{Parameter selection}

$\gamma$ may be considered a parameter of the system: it is dependent on the data acquisition speed, the speed of underlying conductivity changes and the system noise level. By taking covariance on both sides of (8), we have

$$
\Sigma_{\tilde{\mathbf{y}}}=\tilde{\mathbf{J}} \Sigma_{\tilde{\mathbf{x}}} \tilde{\mathbf{J}}^{t}+\Sigma_{\tilde{\mathbf{n}}}
$$

the optimal $\gamma$ is chosen so that the error $\Sigma_{\tilde{\mathbf{y}}}$ $\left(\tilde{\mathbf{J}} \Sigma_{\tilde{\mathbf{x}}} \tilde{\mathbf{J}}^{t}+\Sigma_{\tilde{\mathbf{n}}}\right)$ is minized as

$$
\gamma=\underset{\gamma}{\arg \min }\left\|\Sigma_{\tilde{\mathbf{y}}}-\Sigma_{\tilde{\mathbf{n}}}-\tilde{\mathbf{J}} \Sigma_{\tilde{\mathbf{x}}} \tilde{\mathbf{J}}^{t}\right\|_{F}^{2}
$$

where the subscript $F$ is the Frobenius norm. Since $\Sigma_{\tilde{\mathbf{X}}}=$ $\Gamma \otimes \Sigma_{\mathbf{X}}$ and $\tilde{\mathbf{J}}=\mathbf{I} \otimes \mathbf{J}$, (14) becomes

$$
\gamma=\underset{\gamma}{\arg \min }\left\|\Sigma_{\tilde{\mathbf{y}}}-\Sigma_{\tilde{\mathbf{n}}}-\Gamma \otimes\left(\mathbf{J} \Sigma_{\mathbf{X}} \mathbf{J}^{t}\right)\right\|_{F}^{2}
$$

By taking covariance on both sides of (1), we have

$$
\Sigma_{\mathbf{y}}=\mathbf{J} \Sigma_{\mathbf{X}} \mathbf{J}^{t}+\Sigma_{\mathbf{n}}
$$

so that $\mathbf{J} \Sigma_{\mathbf{X}} \mathbf{J}^{t}=\Sigma_{\mathbf{y}}-\Sigma_{\mathbf{n}}$; we also have $\Sigma_{\tilde{\mathbf{n}}}=\mathbf{I} \otimes \Sigma_{\mathbf{n}}$ and $\Sigma_{\tilde{\mathbf{y}}}=\Gamma_{\mathbf{y}} \otimes \Sigma_{\mathbf{y}}$, where $\Gamma_{\mathbf{y}} \in \mathbb{R}^{(2 d+1) \times(2 d+1)}$ is the correlation matrix of $\tilde{\mathbf{y}}$. Thus the optimal $\gamma$ is calculated by $\gamma=\underset{\gamma}{\arg \min }\left\|\Gamma_{\mathbf{y}} \otimes \Sigma_{\mathbf{y}}-\mathbf{I} \otimes \Sigma_{\mathbf{n}}-\Gamma \otimes\left(\Sigma_{\mathbf{y}}-\Sigma_{\mathbf{n}}\right)\right\|_{F}^{2}$

$\Gamma_{\mathbf{y}}$ and $\Sigma_{\mathbf{y}}$ can be calculated directly from the data. $\Sigma_{\mathbf{n}}$ can be measured by calibration of EIT system.

\section{RESULTS}

Numerical simulations were conducted using a 3-D 10slice cylindrical FEM model with 576 elements each slice (unit radius) using the EIDORS software [7]. Illustrated as figure (1), the model height is $1(z:-0.5 \sim 0.5) ; 2$ electrode rings (8 electrodes each) was attached at $z= \pm 0.2$ using adjacent stimulation and measurement pattern. Inside this model, a conductive sphere with 0.05 unit radius spires upward, moving clockwise (bird's eye view) along a trajectory with radius $2 / 3$ unit, while moving upward from $z=-0.3$ to $z=0.3$. The sphere rotates in the horizontal plane at one rotation per 40 frames. The noise performance of the algorithms was tested by adding pseudo random, zero mean Gaussian noise with the same random seed. Images were reconstructed on a 3-D model with 4-slice and 64 elements each slice, which differs from the simulation model to avoid the inverse crime.

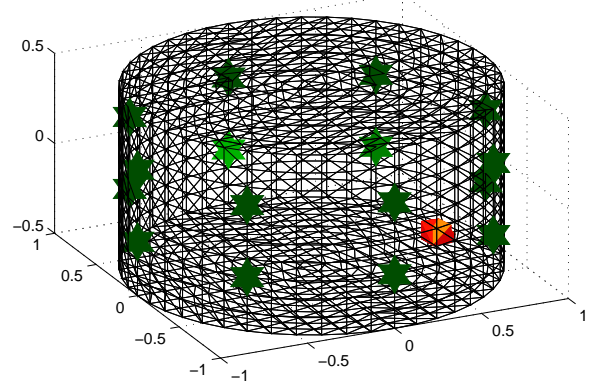

F ig. 1 3-D 10-slice finite element model. Electrodes are indicated by green stars, while the conductive target is shown in red.

Reconstructed targets were calculated for evaluation of three algorithms: GN method; GN method with temporal solver; GN method with 4-D prior. The current data frame was chosen when the sphere located at $(x, y, z)=$ 
$(0.54,-0.39,-0.24)$ (figure 1). For temporal solver, $d=3$ frames were calculated before and after the current frame, respectively. The reconstructions were plotted as slices vertically chosen at $z=0, \pm 0.24$. Figures 2 and 3 show images with Noise to Signal Ratios (NSR) of 0 and 2, respectively. NSR was defined as $\sigma_{\mathbf{n}} / \overline{\mathbf{y}}$. The optimal value of $\gamma$ was calculated based on II-C as 0.86 and 0.76 , for NSR $=0$ and $\mathrm{NSR}=2$, respectively; $\eta$ was heuristically chosen as 0.7 for this reconstruction model.

In order to choose a hyperparameter to allow comparison across algorithms, we select its value for all algorithms in order to give a fixed "Noise Figure" [8]. Due to the fact that when noise figure is high, there is no significant difference between GN method and temporal/4-D prior methods [8], here we only investigate low noise figure cases $(N F=0.1)$.

In order to classify the quality of reconstructed images, the following figures of merit are used: (a) the vertical position of the reconstructed target is correct (matching the location of the phantom target, $z=-0.24$ ); (b) the point spread function (PSF) of the target is small in the horizontal plane; (c) the PSF is small in the vertical plane (this is determined by looking for ghost target regions in the slices at $z=0$ and 0.24 ); (d) the image is not sensitive to measurement noise. Using these criteria and figures 2 and 3, the reconstruction algorithms were ranked in quality order, giving from worst to best: conventional GN, temporal solver, 4-D prior solver.

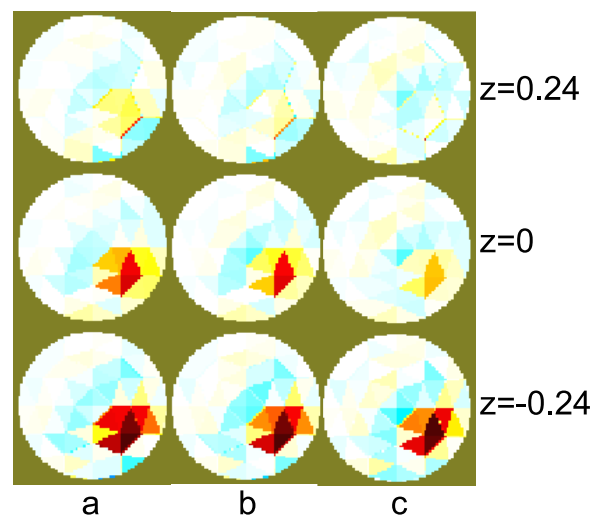

$\mathrm{F}$ ig. 2 Algorithm comparison: NSR=0, NF=0.1 $\gamma=0.86$. (a) conventional GN solver; (b) Temporal solver; (c) 4-D prior solver

\section{CONCLUSION}

A novel EIT reconstruction algorithm is proposed, based on a 4-D regularization prior. By introducing temporal correlations among image frames, the temporal solver is

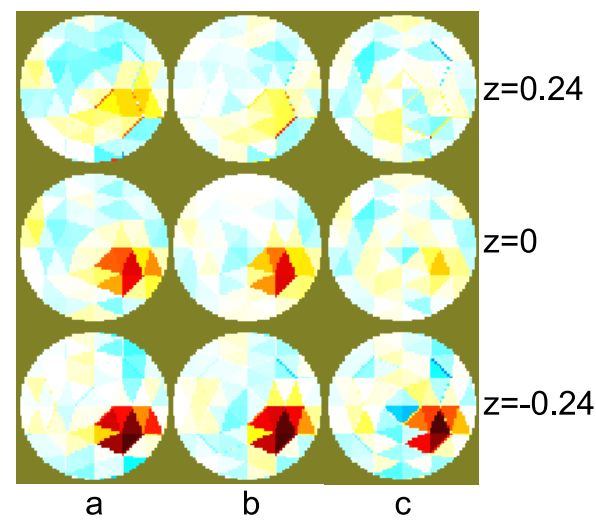

$\mathrm{F}$ ig. 3 Algorithm comparison: $\mathrm{NSR}=2, \mathrm{NF}=0.1 \gamma=0.76$.(a) conventional GN solver; (b) Temporal solver; (c) 4-D prior solver

able to improve reconstructed image quality. With spatial interslice correlation added, the 4-D prior solver improved reconstruction resolution further.

\section{ACKNOWLEDGMENT}

\section{REFERENCES}

1. Wilkinson A J, Randall E W, Cilliers J J, Durrett D R, Naidoo T and Long T (2005) A 1000-measurement frames/second ERT data capture system with real-time visualization, IEEE Sensors Journal 5 300-307

2. Dehgani H, Soni N, Halter R, Hartov A and Paulsen K D (2005) Excitation patterns in three-dimensional electrical impedance tomography, Physiol. Meas. 26 S185-S197

3. Graham B and Adler A (2007) Electrode Placement Configurations for 3D EIT, Physiol. Meas. Mar 2007

4. Vauhkonen P J, Vauhkonen M, Savolainen T and Kaipio J P (1999) Three-dimensional electrical impedance tomography based on the complete electrode model, IEEE Trans, Biomedical Engineering, Vol 46, Issue 9, 1150-1160

5. Vauhkonen M, Karjalainen P A, Kaipio J P (1998) A Kalman filter approach to track fast impedance changes in electrical impedance tomography, IEEE Trans. Biomed. Eng,45 486-493

6. Cheney M, Isaacson D, Newell J C, Simske S and Goble J C (1990) NOSER: an algorithm for solving the inverse conductivity problem, Int.J.Imaging Syst.Technol. 2 66-75

7. Adler A and Lionheart W R B (2006) Uses and abuses of EIDORS: An extensible software base for EIT, Physiol. Meas. 27 S25-S42

8. Adler A, Dai T and Lionheart W R B (2007) Temporal Image Reconstruction in Electrical Impedance Tomography, In press, Physiol. Meas., April 2007

Address of the corresponding author:

Author: Andy Adler

Institute: Carleton University

Street: 1125 Colonel By Drive

City: Ottawa

Country: Canada

Email: adler@sce.carleton.ca 